\title{
Near Optimal Low-hit-zone Frequency Hopping Sequence Set with Partial Hamming Correlation Property
}

\author{
Zheng Xiao, Xiangqian Dong and Li Du \\ Chengdu Neusoft University, Chengdu, 618201, China
}

\begin{abstract}
In this paper, we present a class of low-hit-zone (LHZ) frequency hopping sequence set (LHZ FHS set) with periodic partial Hamming correlation property from the Cartesian product of known FHS sets. It is shown that the sequence set is near optimal with respect to the partial Hamming correlation bound of the LHZ FHS set.
\end{abstract}

Keywords-frequency hopping sequence set; periodic partial Hamming correlation; low hit zone; periodic Hamming correlation

\section{INTRODUCTION}

Frequency hopping spread spectrum and direct sequence spread spectrum are two main spread coding technologies in wireless communication systems. Being anti-jamming, secure, and multiple access properties, Frequency hopping multiple access (FHMA) spread spectrum systems have been found many applications in cellular mobile communication, shortrange wireless communication, personal communication, and so on [1-2]. In practical application systems, frequency hopping sequences (FHSs) are used to specify which frequency will be used for transmission in each time slot. And in multiple access environments, it is important to keep the mutual interference between transmitters at a level as low as possible. When two or more sources transmit the same frequency slot at the same time, mutual interference occurs. The degree of the mutual interferences closely related to the Hamming crosscorrelation properties of the FHSs ([1-3]). So, it is desirable to employ FHSs with low Hamming correlation to reduce the multiple-access interference of frequencies [4]. Thus, it is an important problem that design FHS set with good Hamming correlation properties.

In quasi-synchronous FHMA systems, different from the conventional FHSs, relative delays between district users are restricted within a zone around the origin, so, FHS set with low Hamming correlation within the fixed zone is more useful, this class of sequence set is called LHZ FHS set. The significance of LHZ FHS set is that, even there are relative delays between the transmitted FHSs, the number of hits will be kept at a very low level between different sequences as long as the relative delays do not exceed certain limit, thus reducing or eliminating the mutual interference. Now, several optimal LHZ FHS sets meeting the Peng-Fan-Lee bounds [5] have been covered in the literature [5-11].

Practically, because the limited synchronization time or hardware complexity, so that one often pay more important attentions to the periodic partial Hamming correlation properties (PPHCP) of FHSs, where the Hamming correlation is computed over only subsequences of FHSs. And correlation window length is shorter than the period of the chosen FHSs. Moreover, the window length may vary from time to time depending on the channel conditions. So, FHSs with PPHCP are important for certain application scenarios than the full period Hamming correlation sequences. Up to now, the constructions of this class of FHSs have not yet been popularly reported [12-14].

Furthermore, a LHZ FHS set with PPHCP is called LHZPPHC FHS set. In wireless communication systems, where possess the properties of quasisynchronous FHMA systems and limited synchronization time or hardware complexity, it is necessary to construct LHZ-PPHC FHS set. It is a challenging and interesting problem to study this class of FHS set. Now, very few of LHZ-PPHC FHS sets were covered in the literature. In 2013, Xing Liu et al. [15] constructed a class of LHZ-PPHC FHS set based on interleaving. In 2015, Ch. Y. Wang et al. [16] constructed two classes of LHZ-PPHC FHS set based on the Cartesian product of known FHS sets.

In this paper, we pay particular attentions to the construction of the LHZ-PPHC FHS set. The new construction is based upon the Cartesian product. The rest of this paper is organized in the following manners: In section 2, we review some preliminaries on FHSs, and summarize the key terminologies and notations; In section 3, we construct a new class of LHZ-PPHC FHS set, and verify that the FHS set is near optimal with respect to the Niu-Peng bound [12].

\section{PRELIMINARIES}

Let $F=\left\{f_{1}, f_{2}, \ldots, f_{q}\right\}$ be a frequency slot set with size $q, S$ is a set of $M$ FHSs of length $N$. For any two frequency slots $f_{1}$, $f_{2} \in F$, let

$$
h\left(f_{1}, f_{2}\right)= \begin{cases}1 & \text { if } f_{1}=f_{2} \\ 0 & \text { otherwise }\end{cases}
$$

For any two FHSs $x=\left(x_{0}, x_{1}, \ldots, x_{N-1}\right), y=\left(y_{0}, y_{1}, \ldots, y_{N-1}\right) \in S$, the periodic Hamming correlation $H(x, y ; \tau)$ of $x$ and $y$ at time delay $\tau$ is defined as follow

$$
H_{x, y}(\tau)=\sum_{k=0}^{N-1} h\left(x_{k}, y_{k+\tau}\right) \quad 0 \leq \tau<N
$$


where all operations among the position indices are performed modulo $\mathrm{N}$. The maximum periodic Hamming autocorrelation $\mathrm{H}_{\mathrm{a}}(\mathrm{S})$ and the maximum periodic Hamming crosscorrelation $\mathrm{H}_{\mathrm{c}}(\mathrm{S})$ are defined as follows, respectively:

$$
\begin{aligned}
& H_{a}(S)=\max _{1 \leq \tau<N}\left\{H_{x, x}(\tau) \mid x \in S\right\}, \\
& H_{c}(S)=\max _{0 \leq \tau<N}\left\{H_{x, y}(\tau) \mid x, y \in S, x \neq y\right\} .
\end{aligned}
$$

The maximum periodic Hamming correlation $H_{m}(S)$ is defined as follow

$$
H_{m}(S)=\max \left\{H_{a}(S), H_{c}(S)\right\}
$$

Let integers $H_{L a} \geq 0, H_{L c} \geq 0$.Then the low hit zone $L_{H Z}$, the autocorrelation low hit zone $L_{A H Z}$ and the crosscorrelation low hit zone $L_{C H Z}$ of S are defined as follows, respectively:

$$
\begin{aligned}
& L_{H Z}=\min \left\{L_{A H Z}, L_{C H Z}\right\}, \\
& L_{A H Z}=\max _{0<\tau \leq T}\left\{T \mid H_{x, x}(\tau) \leq H_{L a}, \forall x \in S\right\}, \\
& L_{C H Z}=\max _{0 \leq \tau \leq T}\left\{T \mid H_{x, y}(\tau) \leq H_{L c}, \forall x, y \in S, x \neq y\right\} .
\end{aligned}
$$

An FHS set S with $L_{A H Z}>0$ is called LHZ FHS set.

The periodic partial Hamming correlation between $\mathrm{x}$ and $\mathrm{y}$, for the correlation window length $\mathrm{L}$ starting at $\mathrm{j}$, is defined by

$$
H_{x, y}(j \mid L ; \tau)=\sum_{i=j}^{j+L-1} h\left(x_{i}, y_{i+\tau}\right), 0 \leq \tau<N, 1 \leq L<N .
$$

where all operations among the position indices are performed modulo N. Moreover, $H_{x, y}(j \mid L ; \tau)$ is called the periodic partial Hamming autocorrelation when $\mathrm{x}=\mathrm{y}$, and the periodic partial Hamming crosscorrelation when $x \neq y$. The maximum periodic partial Hamming autocorrelation $P_{S, a}(L)$, the maximum periodic partial Hamming crosscorrelation $P_{S, c}(L)$ and the maximum periodic partial Hamming correlation PS, $\mathrm{m}(\mathrm{L})$ are defined by

$$
\begin{aligned}
& P_{S, a}(L)=\max _{0<\tau<N, 0 \leq j<N}\left\{H_{x, x}(j \mid L ; \tau) \mid x \in S\right\}, \\
& P_{S, c}(L)=\max _{0 \leq \tau<N, 0 \leq j<N}\left\{H_{x, y}(j \mid L ; \tau) \mid x \in S, y \in S, x \neq y\right\}, \\
& P_{S, m}(L)=\max \left\{P_{S, a}(L), P_{S, c}(L)\right\} .
\end{aligned}
$$

For any correlation window length $\mathrm{L}, 1 \leq L<N$, let positive integers $l_{1} \geq 0, l_{1} \geq 0$.Then, the $\mathrm{LHZ}{ }_{\mathrm{PAHZ}}(L)$, the LHZ $L_{\mathrm{PCHZ}}(L)$ and the LHZ $L_{\mathrm{PHZ}}(L)$ are defined as follows:

$$
\begin{aligned}
& L_{\mathrm{PAHZ}}(L)=\max _{\substack{0<\tau \leq T \\
0 \leq j<N}}\left\{T \mid H_{x, y}(j \mid L ; \tau) \leq l_{1}, \forall x \in S\right\}, \\
& L_{\mathrm{PCHZ}}(L)=\max _{\substack{0 \leq \tau \leq T \\
0 \leq j<N}}\left\{T \mid H_{x, y}(j \mid L ; \tau) \leq l_{2}, \forall x, y \in S, x \neq y\right\},
\end{aligned}
$$

$$
L_{\mathrm{PHZ}}(L)=\min \left\{L_{\mathrm{PAHZ}}(L), L_{\mathrm{PCHZ}}(L)\right\} .
$$

In 2010, Niu et al. [17] obtained the lower bound on the periodic partial Hamming correlation properties within the LHZ of LHZ FHS set.

Lemma 3. For any FHS set of M FHSs of length $\mathrm{N}$ and correlation window length $\mathrm{L}$ over a frequency slot set $\mathrm{F}$ with size $\mathrm{q}$, and LP the low hit zone of $\mathrm{S}$ with respect to the constant $\mathrm{P}_{\mathrm{Lm}}$. For any integer $\mathrm{Z}, 1 \leq Z \leq L_{P}$, we have

$$
P_{L m} \geq\left\lceil\frac{(M Z+M-q) L}{(M Z+M-1) q}\right\rceil \text {. }
$$

We call this bound as Niu-Peng bound.

Throughout this paper, we use $(\mathrm{N}, \mathrm{M}, \mathrm{q}, \mathrm{h})$ to denote a set of M FHSs of length $\mathrm{N}$ over an alphabet of size $\mathrm{q}$, and maximum periodic Hamming correlation $\mathrm{h}$, and use $(\mathrm{N}, \mathrm{M}, \mathrm{q}$, $\left.\mathrm{L}_{\mathrm{H}}, \mathrm{h}\right)$ to denote a LHZ FHS set with periodic partial Hamming correlation properties, whose LHZ is $\mathrm{L}_{\mathrm{H}}$ and maximum periodic Hamming correlation is h within LHZ.

Definition 2. Let $\mathrm{S}$ be a LHZ FHS set with parameters $\left(\mathrm{N}, \mathrm{M}, \mathrm{q}, \mathrm{L}_{\mathrm{H}}, \mathrm{h}\right), \mathrm{S}$ is said to be near optimal if $\mathrm{h}-1$ reach the lower bound in (2).

\section{NEAR OPTIMAL LHZ-PPHC FHS SET}

Proposition 1 ([16]). For $l=1,2$ and $i=1,2, \cdots, k$, let $S_{l}^{(i)}=\left\{S_{l}^{(i)}(t)\right\}_{t=0}^{N_{i}-1}$ be an FHS over $F_{i}$, and let $R_{l}=\left\{R_{l}(t)\right\}_{t=0}^{N-1}$ be an FHS over $F_{1} \times \cdots \times F_{k}$, defined by

$$
R_{l}(t)=\left(S_{l}^{(1)}(t), \ldots, S_{l}^{(k)}(t)\right)
$$

where $N=\operatorname{lcm}\left(N_{1}, \cdots, N_{k}\right)$, then

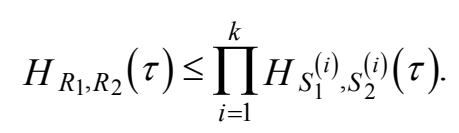


For any $0 \leq \tau \leq N-1$. In particular, for any $1 \leq i_{1} \neq i_{2} \leq k$, $\operatorname{gcd}\left(N_{i_{1}}, N_{i_{2}}\right)=1$, we have

$$
H_{R_{1}, R_{2}}(\tau)=\prod_{i=1}^{k} H_{S_{1}^{(i)}, S_{2}^{(i)}}(\tau)
$$

Theorem 1 ([16]). For $1 \leq i \leq k$, let $X_{i}=\left\{x_{i, l} \mid 0 \leq l \leq L_{i}-1\right\}$ be an FHS set $\left(N_{i}, L_{i}, M_{i} ; \lambda_{i}\right)$ over $\mathrm{F}_{\mathrm{i}}$ satisfying

1). For $0 \leq l \neq m \leq L_{i}-1, H_{x_{i, l}, x_{i, m}}(0)=0$.

2). $N_{1} \neq \operatorname{gcd}\left(\operatorname{lcm}\left(N_{2}, \cdots, N_{k}\right), N_{1}\right)$.

Let $N=\operatorname{lcm}\left(N_{1}, \ldots, N_{k}\right)$. And an FHS set over $F_{1} \times \cdots \times F_{k}$ is defined by

$$
\left\{\begin{array}{l}
X=\left\{x_{i_{1}, \ldots, i_{k}}(t) \mid 0 \leq i_{1} \leq L_{1}-1, \ldots, 0 \leq i_{k} \leq L_{k}-1,0 \leq t \leq N-1\right\}, \\
x_{i_{1}, \ldots, i_{k}}(t)=\left(x_{1, i_{1}}(t), \ldots, x_{k, i_{k}}(t)\right) .
\end{array}\right.
$$$$
\text { Let } L=L_{1} \cdots L_{k}, M=M_{1} \cdots M_{k} \text {, and } \lambda=\lambda_{1} \cdots \lambda_{k} \text {.The }
$$
set $\mathrm{X}$ is a LHZ FHS set with parameters $\left(N, L, M, N_{1}-1, \lambda\right)$.

Construction A: Step1: Let $q$ be a prime power, $0 \leq i<q$, and $X=\left\{x_{i}(t)\right\}_{t=0}^{q-2}$ be an FHS set over $F_{q}$ with parameters $(q-1, q, q, 1)$.And satisfies

$$
H_{x_{l_{0}}, x_{l_{1}}}(0)=0 \quad \text { for } 0 \leq l_{0} \neq l_{1}<q
$$

Step2: Let $p$ be a prime and $q<p-1, \operatorname{gcd}(q-1, p)=1$, $0 \leq j<p-1, \quad Y=\left\{y_{j}(t)\right\}_{t=0}^{p-1}$ be an FHS set over $F_{p}$ with parameters $(p, p-1, p, 1)$.

Step3: Defined the FHS set W over $F_{q} \times F_{p}$ as follow

$$
\left\{\begin{array}{l}
W=\left\{w_{i_{0}, i_{1}}(t) \mid 0 \leq t<(q-1) p\right\} \\
w_{i_{0}, i_{1}}(t)=\left(x_{i_{0}}(t), y_{i_{1}}(t)\right) \\
0 \leq i_{0}<q, 0 \leq i_{1}<p-1
\end{array}\right.
$$

we select q sequences from $W$ to form an FHS set $S$, where the subscript ${ }^{i_{1}}$ isn't constant and the subscript $i_{0}$ take difference values between 0 and $\mathrm{q}-1$, we have

Theorem 1. With respect to the Niu-Peng bound (2), the FHS set $S$ is a near optimal LHZ FHS set with parameters $((q-1) p, q, p q, p-1,1)$

Proof: If ${ }^{i_{1}}$ isn't constant, by definition of the maximum periodic Hamming correlation, we have

$$
\begin{aligned}
H_{\left(i_{0}, i_{1}\right),\left(i^{\prime}, i^{\prime} 1\right)}(\tau)=\sum_{t_{1}=0}^{q-2} h\left(X_{i_{0}}\left(t_{1}\right), X_{i^{\prime} 0}\left(t_{1}+\tau\right)\right) \\
\cdot \sum_{t_{2}=0}^{p-1} h\left(Y_{i_{1}}\left(t_{2}\right), Y_{i^{\prime} 1}\left(t_{2}+\tau\right)\right) .
\end{aligned}
$$

We obtain

$$
H_{a}(S)=0 \quad \text { if } i_{1}=i^{\prime}
$$

And

$H_{c}(S)=\left\{\begin{array}{l}0 \text { if } \tau \equiv 0 \bmod (q-1), \\ p \text { if } \tau \equiv 0 \bmod p, \tau \neq 0 \bmod (q-1) \text { and } i_{1}=i^{\prime}{ }_{1} \\ 0 \text { if } \tau \neq 0 \bmod p, i_{1}=i_{1}^{\prime}{ }_{1}, \\ 1 \quad \text { otherwise. }\end{array}\right.$

So, we have

$$
H_{m}(S)=1 \quad \text { if } \tau<p .
$$

Furthermore, when $0 \leq \tau<p$, we can obtain

$$
P_{m}(L)=\left\lceil\frac{L}{(q-1) p}\right\rceil \text { if } 1 \leq L \leq(q-1) p .
$$

According to the Niu-Peng bound (2), we obtain

$$
P_{m}(L) \geq\left\lceil\frac{(M Z+M-q) L}{(M Z+M-1) q}\right\rceil=\left\lceil\left(\frac{p q-p q}{p q-1}\right) \cdot \frac{L}{p q}\right\rceil=0
$$

It is obvious that $S$ is near optimal for any correlation window.

Lemma 7 (Solomon, [18]): Let $p$ be a prime, $0 \neq \beta_{r} \in G F(p), \quad a_{r}=\left\{a_{k}^{(r)}=k \beta_{r} \mid k=0,1, \cdots, p-1\right\}$ be an FHS 
over $G F(p), 0 \leq r<p$,the FHS set given by

$$
A=\left\{a_{r} \mid 1 \leq r \leq p-1\right\} .
$$

Then

$$
H_{A_{i}, A_{j}}(\tau)=\left\{\begin{array}{cc}
p & \text { if } \tau \equiv 0 \bmod p \text { and } i=j \\
0 & \text { if } \tau \neq 0 \bmod p \text { and } i=j \\
1 & \text { otherwise }
\end{array}\right.
$$

Lemma 6 (Titlebaum, [19]): Let q be a prime power and $\alpha$ a primitive element of $\mathrm{F}_{\mathrm{q}}$. Assume that $F_{q}=\left\{\zeta_{i} \mid 0 \leq i<q\right\}$, for $0 \leq i<q, X=\left\{X_{i}(t)\right\}_{t=0}^{q-2}$ be an FHS set over $\mathrm{F}_{\mathrm{q}}$, given by

$$
X_{i}(t)=\alpha^{t}+\zeta_{i}
$$

Then

$$
H_{X_{i}, X_{j}}(\tau)=\left\{\begin{array}{lc}
q-1 & \text { if } \tau \equiv 0 \bmod (q-1) \text { and } i=j \\
0 & \text { if } \tau \neq 0 \bmod (q-1) \text { and } i=j \\
0 & \text { if } \tau \equiv 0 \bmod (q-1) \text { and } i \neq j \\
1 & \text { otherwise. }
\end{array}\right.
$$

The follow example comes from the product of Solomon's FHS set and Titlebaum's FHS set.

Example: Let $\mathrm{p}=31$, we construct a Titlebaum's set as follows:

$$
\begin{gathered}
Z_{0}=\{0,10,20,30,9,19,29,8,18,28,7,17 \ldots\} \\
Z_{1}=\{0,11,22,2,13,24,4,15,26,6,17,28 \ldots\} \\
Z_{2}=\{0,12,24,5,17,29,10,22,3,15,27,8 \ldots\} \\
\ldots \\
Z_{30}=\{0,30,29,28,27,26,25,24,23,22,21 \ldots\}
\end{gathered}
$$

Let $\mathrm{q}=7$, we construct a Solomon's set as follows:

$$
\begin{aligned}
& Z_{0}=\{1,3,2,6,4,5\} ; Z_{1}=\{2,4,3,0,5,6\} ; \\
& Z_{2}=\{3,5,4,1,6,0\} ; Z_{3}=\{4,6,5,2,0,1\} ;
\end{aligned}
$$

By the Chinese remainder theorem, we denote the frequency slot taking an integer instead of order pair of Cartesian product. By applying Construction A, we obtain:

$$
\begin{gathered}
S=\left\{s_{0}=\{32,157,189,159,160,37,193,101,133,103,104 \ldots\}\right. \\
s_{1}=\{187,95,127,97,98,192,131,39,71,41,42,136,75 \ldots\}
\end{gathered}
$$

$$
s_{2}=\{125,33,65,35,36,130,69,194,9,196,197,74,13 \ldots\}
$$

$$
\left.s_{6}=\{157,128,6,39,103,43,45,16,111,144,208,148 \ldots\}\right\} .
$$

when $0 \leq \tau \leq 30$, the maximum periodic Hamming correlation $H_{m}(S)=1$.And for the correlation window length $\mathrm{L}$, it can be verified that the maximum periodic partial Hamming correlation of $S$ is given by

$$
P_{m}(L)=\left\lceil\frac{L}{186}\right\rceil 1 \leq L<186
$$

One can easily check that the hopping sequence set $S$ is near optimal with respect to the Niu-Peng bound (2).

\section{CONCLUSIONS}

In some wireless communication system, the LHZ-PPHC FHS set is used to eliminate MA interference. It is important to construct the LHZ-PPHC FHS set. In this correspondence, a near optimal LHZ-PPHC FHS set is constructed based on the Cartesian product, it may be a challenging problem to find more classes of optimal LHZ-PPHC FHS sets, which are not involved in this paper.

\section{REFERENCES}

[1] P.Z.Fan. M. Darnell. Sequence Design for Communications Applications. Research Studies Press (RSP), Wiley, London (1996).

[2] S. W. Golomb. G. Gong. Signal Design for Good Correlation: For Wireless Communication, Cryptography and Radar. Signal Design for Good Correlation: For Wireless Communication, Cryptography and Radar (2005).

[3] M. K. Simon.J. K. Omura.R. A. Scholtz. B. K. Levitt. Spread Spectrum Communications Handbook, (Revised Ed.). McGraw-Hill Inc (1994).

[4] A. Lempel.H. Greenberger. Families of sequences with optimal Hamming correlation properties, IEEE Transactions on Information Theory, vol.20, no.1, 1974,pp. 90-94.

[5] D. Peng.P. Fan.M. H. Lee. Lower bounds on the periodic Hamming correlations of freq 2, 2006,pp. 208-218.

[6] J.H. Chung.K. Yang. Low-hit-zone frequency-hopping sequence sets with new parameters, Lecture Notes in Computer Science, vol.7280, Sequences and Their Applications(SETA 2012), Waterloo, ON, Canada, June 4-8, 2012, pp. 202-211.

[7] J.H. Chung.K.Yang. New Classes of Optimal Low-Hit-Zone FrequencyHopping Sequence Sets by Cartesian Product, IEEE Transactions on Information Theory, vol.59,no.1, 2013, pp. 726-732.

[8] W. Ma. S. Sun.New designs of frequency hopping sequences with low hit zone, Des.Codes Crypt.vol.60, no.2, 2011,pp. 145-153.

[9] Xinhua Niu.Daiyuan Peng.Zhengchun Zhou.New classes of optimal LHZ FHS with new parameters, The Sixth International Workshop on Signal Design and Its Applications in Communications (IWSDA'11), Guilin, China, October 10-14, 2011,pp. 111-114.

[10] X. Niu.D.Y. Peng.Z.C. Zhou. New classes of optimal frequency hopping sequences with low hit zone, Adv. Math. Communi, vol.7, no.2. 2013, pp. 123-142

[11] X.H. Niu.D.Y. Peng.Z.C. Zhou. New classes of optimal low hit zone frequency-hopping sequences with new parameters by interleaving technique, IEICE Transactions on Fundamentals, vol.55, no.11, 2012,pp. 1835-1842. 
[12] X.H. Niu.D.Y. Peng.Z.C. Zhou.Frequency/time hopping sequence sets with optimal partial Hamming correlation properties, Science China (Information Series), vol.55, no.10, 2012,pp. 2207-2215.

[13] Z.C. Zhou.X.H. Tang.X.H. Niu.P. Udaya. New classes of frequencyhopping sequences with optimal partial correlation, IEEE Trans. Inform. Theory, vol.58, no.1, 2012,pp. 453-458.

[14] Y. C. Eun.S. Y. Jin.Y. P. Hong.H. Y. Song. Frequency Hopping Sequences With Optimal Partial Auto-correlation Properties, IEEE Trans. Inf. Theory, Vol.50, No.10, 2004,pp.2438-2442, October.

[15] Xing Liu.Daiyuan Peng.Hongyu Han. Low-hit-zone frequency hopping sequence sets with optimal partial Hamming correlation properties, Des. Codes Crypt, 2013, pp. 153-164.

[16] Ch. Y. Wang, D. Y. Peng, H. Y. Han, L. M. L. Zhou. New sets of low-hit-zone frequency-hopping sequence with optimal maximum periodic partial Hamming correlation, Science china Information Sciences, vol. 58, no. 12, 2015,pp. 1-15.

[17] Xinhua Niu.Daiyuan Peng.Fang Liu.Xing Liu.Lower Bounds on the Maximum Partial Correlations of Frequency Hopping Sequence Set with Low Hit Zone, IEICE Trans.Fundamentals, vol.E93-A, no.11,2010,pp. 2227-2231.

[18] Titlebaum E L.Time-Frequency Hop Signals Part :Coding Based Upon the Theory of Linear Congruences. IEEE Trans.AES-17(4), 1981,pp. 490-493.

[19] G. Solomon.Optimal frequency hopping for multiple accesses Proc.1977 Symp. Spread Spectrum Commun, San Diego, CA, USA, Mar. 13-16, 1977,pp. 33-35. 